

University of Pennsylvania ScholarlyCommons

December 2002

\title{
Preferred orientation in fibers of HiPco single wall carbon nanotubes from diffuse $x$-ray scattering
}

Wei Zhou

University of Pennsylvania, wzhou@seas.upenn.edu

Karen I. Winey

University of Pennsylvania, winey@lrsm.upenn.edu

John E. Fischer

University of Pennsylvania, fischer@seas.upenn.edu

Sivarajan Ramesh

Rice University

Rajesh K. Saini

Rice University

See next page for additional authors

Follow this and additional works at: https://repository.upenn.edu/mse_papers

\section{Recommended Citation}

Zhou, W., Winey, K. I., Fischer, J. E., Ramesh, S., Saini, R. K., Ericson, L. M., Davis, V. A., Pasquali, M., Hauge, R. H., \& Smalley, R. E. (2002). Preferred orientation in fibers of HiPco single wall carbon nanotubes from diffuse $x$-ray scattering. Retrieved from https://repository.upenn.edu/mse_papers/23

Copyright Materials Research Society. Reprinted from MRS Proceedings Volume 740.

2002 Fall Meeting Symposium I

Nanomaterials for Structural Applications

Publisher URL:http://www.mrs.org/members/proceedings/fall2002/i/l12_21.pdf

This paper is posted at ScholarlyCommons. https://repository.upenn.edu/mse_papers/23

For more information, please contact repository@pobox.upenn.edu. 


\title{
Preferred orientation in fibers of HiPco single wall carbon nanotubes from diffuse x-ray scattering
}

\author{
Abstract \\ Neat Fibers of HiPco single wall carbon nanotubes extruded from strong acid suspensions exhibit \\ preferred orientation along fiber axes. We characterize the extrusion-induced alignment using $\mathrm{x}$-ray fiber \\ diagrams and polarized Raman scattering, using a model which allows for some fraction of the sample to \\ remain completely unaligned. We show that both x-ray and Raman data are required for a complete \\ texture analysis of SWNT fibers.

\section{Comments} \\ Copyright Materials Research Society. Reprinted from MRS Proceedings Volume 740. \\ 2002 Fall Meeting Symposium I \\ Nanomaterials for Structural Applications \\ Publisher URL:http://www.mrs.org/members/proceedings/fall2002/i/I12_21.pdf

\section{Author(s)} \\ Wei Zhou, Karen I. Winey, John E. Fischer, Sivarajan Ramesh, Rajesh K. Saini, Lars M. Ericson, Virginia A. \\ Davis, M. Pasquali, Robert H. Hauge, and Richard E. Smalley
}




\title{
PREFERRED ORIENTATION IN FIBERS OF HIPCO SINGLE WALL CARBON NANOTUBES FROM DIFFUSE X-RAY SCATTERING
}

W. Zhou, K. I. Winey, J. E. Fischer

Department of Materials Science and Engineering, University of Pennsylvania, Philadelphia PA; S. Ramesh, R. K. Saini, L. M. Ericson, V. A. Davis, M. Pasquali, R. H. Hauge, R. E. Smalley Center for Nanoscale Science and Technology, Rice University, Houston TX.

\begin{abstract}
Neat Fibers of HiPco single wall carbon nanotubes extruded from strong acid suspensions exhibit preferred orientation along fiber axes. We characterize the extrusion-induced alignment using x-ray fiber diagrams and polarized Raman scattering, using a model which allows for some fraction of the sample to remain completely unaligned. We show that both x-ray and Raman data are required for a complete texture analysis of SWNT fibers.
\end{abstract}

\section{INTRODUCTION}

Macroscopic oriented arrays of single wall carbon nanotubes (SWNT) [1-4] could be the starting point for the construction of useful structures which maintain a high degree of the excellent axial properties expected from perfect SWNT. Such nanotubes produced by the HiPco process [5] offer promise for high strength, light weight, electrically conducting structural elements at lower cost than other nanotube forms. The properties of HiPco fibers will depend on the degree of SWNT alignment [6]. In this paper we study the preferred orientation by combining diffuse $x$-ray and polarized Raman scattering [7].

\section{EXPERIMENTAL DETAILS}

Fibers were produced from purified HiPco SWNT [8] containing less than 1 at.\% residual metal catalyst. Nanotubes were mixed with oleum or $100 \%$ sulfuric acid, concentrations up to 8 wt. $\%$, at $\sim 110^{\circ} \mathrm{C}$ for 24 hours with constant argon flow to eliminate moisture. Mixing at low concentration was achieved by a low shear magnetic stirring while medium shear double helix mixer was used for high concentrations. Fibers were then extruded into a coagulation bath using a syringe with no drawing applied. Different diameter fibers were produced by using syringe needles with different inside diameters. Detailed description can be found elsewhere [9].

Texture analysis was done on 3 fibers, all produced from HiPco batch 93 (purified) under different experimental conditions. HPR93a was extruded from $8 \%$ SWNT in 100\% sulfuric acid through a 500 micron syringe needle; HPR $93 \mathrm{~b}$ was $6 \%$ SWNT through a 125 micron syringe; HPR93c was $6 \%$ SWNT through a 250 micron syringe. No mechanical stretching was applied during or after coagulation. The diameters of 3 fibers were 220,60 and $110 \mu \mathrm{m}$ respectively, about a factor of two smaller than orifice due to collapse of the gel state. The nanotubes in neat fibers are heavily p-doped by sulfuric acid. To measure the intrinsic properties we annealed neat fibers either in flowing argon at $1100^{\circ} \mathrm{C}$ for 24 hours or in vacuum at $1150^{\circ} \mathrm{C}$ for 2 hours using a slow temperature ramp [10]. Most acid residue and some amorphous carbon were expected to be removed in the annealing process. 
Texture analysis was done on neat and annealed fibers by combining x-ray diffuse and polarized Raman scattering. X-ray scattering was performed on a multi-angle diffractometer equipped with $\mathrm{Cu}$ rotating anode, double-focusing optics, evacuated flight path and 2-D wire detector. All samples were measured in transmission for 2 hours. For large diameter fibers, a single piece gave enough signal; for small diameters several pieces were carefully assembled parallel to each other. Polarized Raman measurements were done in VV geometry on a Renishaw Ramanscope 1000 system using 514.4 nm excitation. Both x-ray and Raman data were analyzed with a "2-phase" model described here, to account for both aligned and unaligned SWNT.

\section{RESULTS AND DISCUSSION}

HiPco SWNT exhibit weak or no x-ray Bragg intensity due to the broad diameter distribution [11]. Our fibers of HiPco also exhibit poor crystallinity, and the x-ray scattering in the wide angle region $(\mathrm{Q}>0.1)$ is made up of diffuse scattering from isolated tubes and poorly crystallized large bundles, plus the low Q tail of small-angle x-ray scattering (SAXS) from uncorrelated pores, impurity particles etc. The SWNT-related diffuse scattering should in principle follow the Bessel function form factor of a cylindrical shell of charge [12], but the oscillations are smeared out and we observe monotonically decreasing intensity with increasing Q which cannot be separated from non-SWNT-related scattering. On the other hand it is reasonable to assume that only the SWNTs contribute to the observed anisotropy. Therefore we can obtain reliable distribution widths that characterize the aligned SWNTs, but we learn nothing about unaligned SWNTs. In previous studies the on fibers or mats diffuse scattering was classified with the sample-independent background and only the weak Bragg intensity was considered [3].

X-ray profiles (obtained by azimuthal integration of the 2D data) from neat HPR93a fibers are shown in Fig.1. No Bragg peaks were detected from the neat fiber; the broad peak at $Q$ $\sim 1.6 \mathrm{~A}^{-1}$ is most likely due to acid residues since amorphous carbon is effectively removed by the purification scheme. After vacuum annealing we observe stronger low-Q scattering, 3 weak Bragg peaks near $0.45,0.75$ and $1.1 \mathrm{~A}^{-1}$, and the disappearance of the broad peak at $1.6 \mathrm{~A}^{-1}$. We attribute these changes to removal of acid residues and partial reorganization of tubes within bundles. Although vacuum annealing improved the crystallinity of nanotubes to some extent, the main contribution to SWNT scattering remains diffuse.

From the 2D data sets, we take sectors along the radial $\mathrm{Q}$ direction out of $1^{\mathrm{O}}$ wedges and plot their intensity vs. azimuthal angle $\chi$. Preferred orientation is then deduced in the range of $0.35<\mathrm{Q}<0.55$. In Fig.2(a) we show the result from the neat fiber HPR93c. The solid curve is the least squares fit to Gaussians centered near $\chi=0^{\circ}$ and $180^{\circ}$ plus a constant, where the fiber axis is perpendicular to these maxima. The constant corresponds to isotropic scattering from both non-SWNT constituents and unaligned tubes. The Gaussians are fully attributed to nanotube alignment. We term this analysis the "2-phase" model. The fitted Gaussian FWHMs are $63^{\circ}$, $55^{\circ}$ and $45^{\circ}$ for neat fibers $a, c$ and $b$ respectively, where $b$ was spun from $6 \%$ SWNT through the $125 \mu \mathrm{m}$ orifice. Similar results were obtained from the annealed counterparts. Fitted values are collected in Table I. 
Texture is also revealed in the small angle diffuse scattering. Figure 2(b) shows the azimuthal dependence of the Q-integrated intensity from 0.035 to $0.070 \mathrm{~A}^{-1}$ for annealed sample c. The corresponding FWHM, 58 ${ }^{\circ}$, was only slightly larger than the high $\mathrm{Q}$ result. Since the scattering bodies are rod-like nanotube bundles or aggregates, we assign the anisotropic SAXS to the preferred orientation along the fiber axis of these rod-like objects.

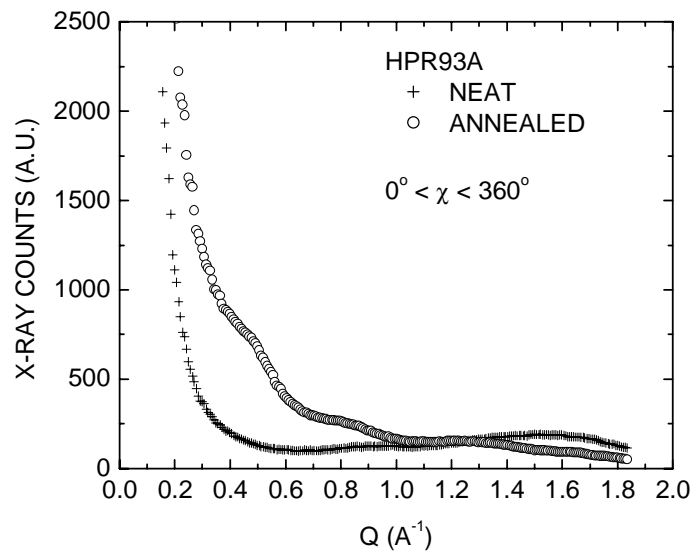

a)

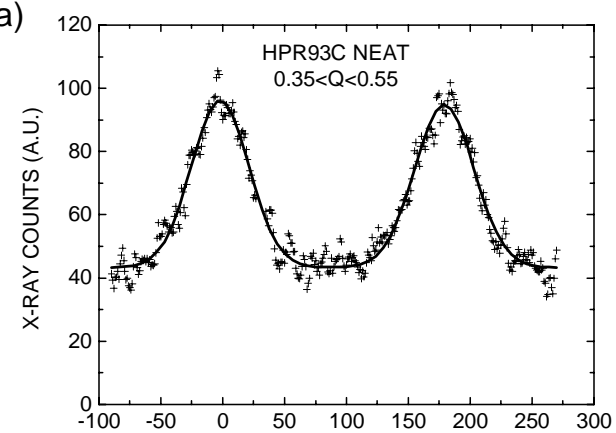

b)

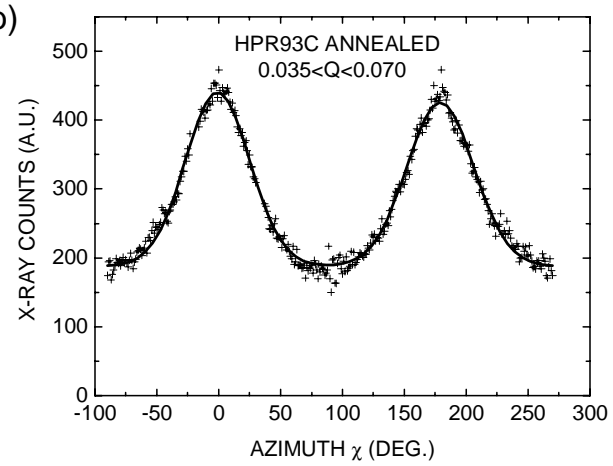

Figure 1. X-ray scattering from neat and annealed fiber HPR93a. Samples are in transmission geometry. Profiles are obtained by azimuthal integration of the $2 \mathrm{D}$ data.
Figure 2. Background-subtracted X-ray counts, summed over certain $Q$ intervals, every $1^{\circ}$ in $\chi$. Data are the symbols; fits to two Gaussians plus a constant are the smooth curves. Top: neat HPR93c; Bottom: annealed HPR93c.

Raman measurements using VV polarization were carried out at many angles $\Psi$ between the fiber axis and polarization vector, to obtain a characteristic distribution analogous to x-ray fiber diagrams $[6,10]$. We used the same "2-phase" model as for our x-ray analysis. The fibers are axially symmetric so the distribution function has cylindrical symmetry. We accounted for 
anisotropic optical attenuation by the nanotubes; $f_{\mathrm{abs}} \propto 1 /(\cos \phi+\mathrm{K} \sin \phi)$ where $\phi$ is the angle between polarization vector and nanotube axis and $\mathrm{K}=\alpha_{\perp} / \alpha_{\|}\left(\alpha_{\|}\right.$and $\alpha_{\perp}$ are the components of polarized absorption coefficients) [3]. It is believed that $K$ is between 0 and $1 / 4$ for the wavelength of interest. In principle, both the aligned fraction A and FWHM are obtained by fitting the deviation from a $f_{\mathrm{abs}} \cos ^{4} \Psi$ law for $100 \%$ perfectly aligned tubes. Due to the large error bar of the Raman data, it's almost impossible to reliably fit A and FWHM simultaneously. Thus, we input the FWHM from the X-ray analysis and perform a one-parameter fit to the Raman data that optimizes A.

Peak intensities of the tangential Raman $\mathrm{G}_{2}$-band at $1590 \mathrm{~cm}^{-1}$ were recorded from 3 different $2 \mu \mathrm{m}$ spots to account for inhomogeneity in the fiber, at each of $7 \Psi$ values. Analysis is shown in Fig.3. Least squares fits assuming $\mathrm{K}=1 / 8$ show that the aligned fractions for neat HPR93 a, c and b are $0.83,0.90$ and 0.94 respectively. Slightly smaller or larger values result from assuming $K=0$ and $1 / 4$ respectively, from which we estimate the error on $A$ to be \pm 0.02 . Note that A decreases with decreasing orifice diameter. An obvious explanation is that smaller orifices do a better job of excluding or breaking up the undispersed aggregates. Small increases in A were observed after annealing, Table I.

From Raman spectra, it is also found that the shapes of the RBM band and G band are quite different for neat and annealed fibers, as shown in Fig.4. This is mainly because neat and annealed samples are under different resonance conditions. The neat fibers are heavily p-doped and the doping shifts the Fermi energy, thus certain tubes in neat fibers lost Raman resonance. The annealing process at high temperature de-doped the nanotubes so that the Raman spectra of annealed fibers resemble those of ordinary HiPco materials [11].

\section{SUMMARY}

HiPco fibers produced by simply extruding a strong acid suspension of SWNT exhibit moderate nanotube alignment. Further improvements may be expected by applying additional extensional flow or stretching in the gel state [13]. Structural analysis by combining x-ray and Raman scattering unambiguously shows that more dilute SWNT suspension and smaller diameter syringe needle generally result in fibers with better alignment. The synthesis parameters and texture analysis fit parameters for both neat and annealed fibers are summarized in Table I. For the first time, we show that fiber diagrams can be obtained from diffuse X-ray scattering of nanotubes. We also show that a complete fiber texture can be determined by combining x-ray fiber diagram and Polarized Raman scattering, using a "2-phase" model which allows for a fraction of the sample to remain completely unaligned. Results of this analysis are used to optimize the fiber process. Correlation between texture and anisotropic thermal and electrical properties will be reported elsewhere.

\section{ACKNOWLEDGEMENTS}

This research was supported by the Office of Naval Research Grant No. N000140010720 and N000140010657. 


$$
\text { a) }
$$
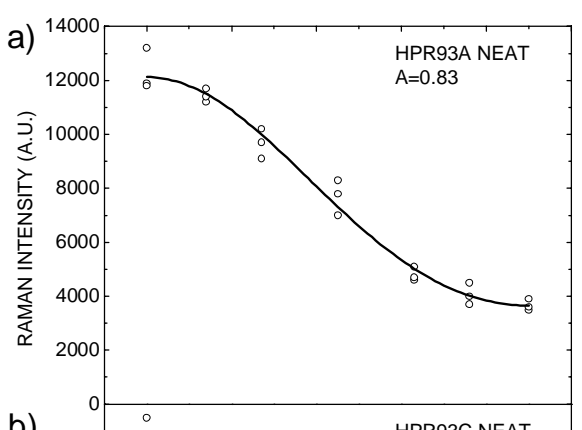

b)
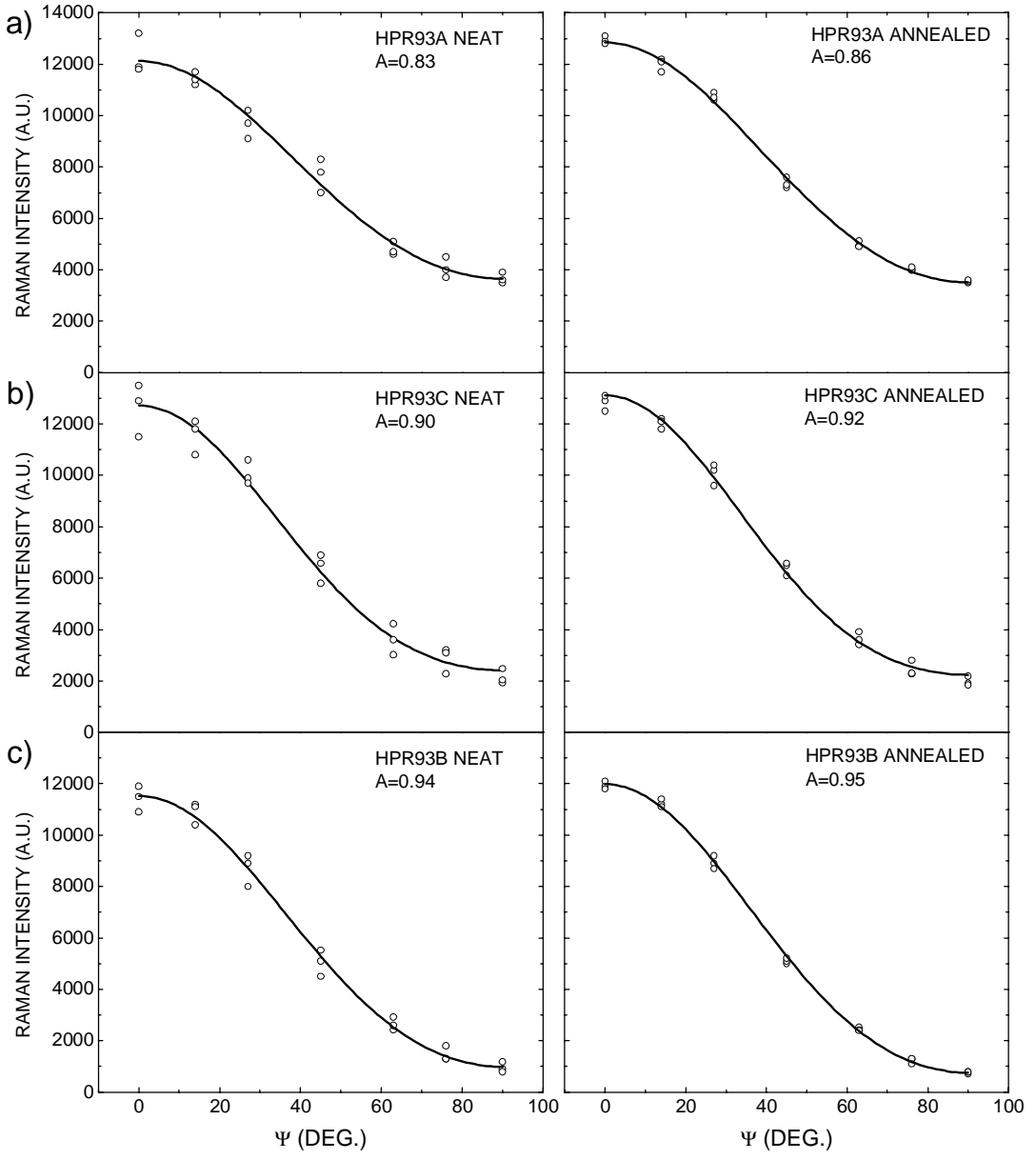

Figure 3. Analysis of polarized Raman spectra. Symboles are Raman G2-band intensities, measured on 3 spots on the sample, versus the angle between polarization and fiber axis for HPR93A (top), C (middle) and $\mathrm{B}$ (bottom). Solid curves are least square fits to the model described in the text, with fitted aligned fraction indicated in the figures.

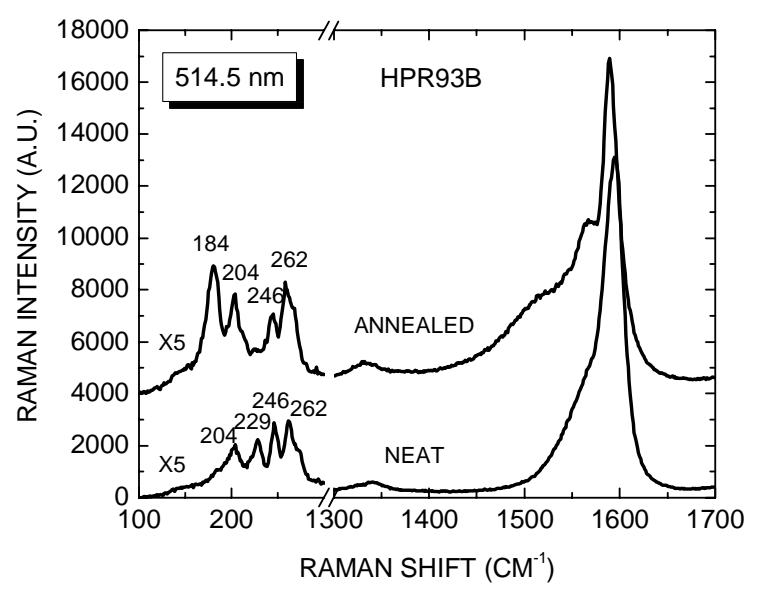

Figure 4. Raman spectra of neat and annealed fiber HPR93B. Note the difference of both RBM band and $\mathrm{G}$ band between neat and annealed fibers. 
Table I. Summary of the synthesis parameters, texture analysis fit parameters for neat and annealed HiPco fibers.

\begin{tabular}{|l|l|l|l|l|l|l|}
\hline & \multicolumn{2}{|c|}{ HPR93A } & \multicolumn{2}{c|}{ HPR93C } & \multicolumn{2}{c|}{ HPR93B } \\
\hline Concentration & \multicolumn{2}{|c|}{8 wt.\% } & \multicolumn{3}{c|}{6 wt.\% } & \multicolumn{2}{c|}{6 wt.\% } \\
\hline Orifice $(\mu \mathbf{m})$ & \multicolumn{2}{|c|}{500} & \multicolumn{2}{c|}{250} & \multicolumn{2}{c|}{125} \\
\hline & Neat & Annealed & Neat & Annealed & Neat & Annealed \\
\hline FWHM(deg.) & 63 & 64 & 55 & 54 & 45 & 43 \\
\hline $\begin{array}{l}\text { Aligned Fraction, } \\
\text { A ( } \pm \text { 0.02) }\end{array}$ & 0.83 & 0.86 & 0.90 & 0.92 & 0.94 & 0.95 \\
\hline
\end{tabular}

\section{REFERENCES}

1. L. Jin. C. Bower and O. Zhou, Appl. Phys. Lett. 3, 1197 (1998).

2. J. C. Hone, M. C. Llaguno, N. M. Nemes, J. E. Fischer, D. E. Walters, M. J. Casavant, J. Schmidt and R. E. Smalley, Appl. Phys. Lett. 77, 666 (2000).

3. P. Launois, A. Marucci, B. Vigolo, A. Derre and P. Poulin, J. Nanoscience and Nanotechnology Vol. 1 (2001) pp125-128.

4. J. E. Fischer, W. Zhou, J. Vavro, M. C. Llaguno, C. Guthy, R. Haggenmueller, K. I. Winey, M. J. Casavant and R. E. Smalley, J. Appl. Phys. (Submitted)

5. Michael J. Bronikowski, Peter A. Willis, Daniel T. Colbert, K. A. Smith, and Richard E. Smalley, J. Vac. Sci. Technol. A 19, 1800 (2001)

6. R. Haggenmueller, H. H. Gommans, A. G. Rinzler, J. E. Fischer and K. I. Winey, Chem. Phys. Lett. 330, 219 (2000).

7. H. H. Gommans, J. W. Alldredge, H. Tashiro, J. Park, J. Magnuson and A. G. Rinzler, J. Appl. Phys. 88, (2000) 2509.

8. I. W. Chiang, B. E. Brinson, A. Y. Huang, P. A. Willis, M. J. Bronikowski, J. L. Margrave, R. E. Smalley, and R. H. Hauge, J. Phys. Chem. B, 105(35), 8297 (2001)

9. Virginia A. Davis, Lars M. Ericson, Rajesh Saini, Ramesh Sivarajan, R. H. Hauge, Richard E. Smalley and Matteo Pasquali, AIChE proceedings (2001)

10. A. G. Rinzler, J. Liu, P. Nikolaev, C. B. Huffman, F. J. Rodriguez-Macias, P. J. Boul, A. H. Lu, D. Heymann, D. T. Colbert, R. S. Lee, J. E. Fischer, A. M. Rao, P. C. Eklund, and R. E. Smalley, Applied Physics A 67, 29 (1998).

11. W. Zhou, Y. H. Ooi, R. Russo, P. Papanek, D. E. Luzzi, J. E. Fischer, M. J. Bronikowski, P. A. Willis and R. E. Smalley, Chem. Phys. Lett. 350, 6 (2001).

12. A. Thess, R. Lee, P. Nikolaev, H. Dai, P. Petit, J. Robert, C. Xu, H. Lee, S.G. Kim, D. T. Colbert, G. Scuseria, D. Tomanek, J. E. Fischer and R. E, Smalley, Science 273, 483 (1996).

13. Brigitte Vigolo, Pascale Launois, Marcel Lucas, Stéphane Badaire, Patrick Bernier, and Philippe Poulin, Mat.Res.Soc.Symp.Proc., 706, Z1.4.1 (2002) 